\title{
Oral Vaccines-Types, Delivery Strategies, Current and Future Perspectives
}

\author{
Mohammad Nazmul Hasan*1 and S M Shatil Shahriar ${ }^{2}$ \\ ${ }^{1}$ Department of Green Bioengineering, Korea National University of Transportation, Korea \\ ${ }^{2}$ Department of Chemical and Biological Engineering, Korea National University of Transportation, Korea
}

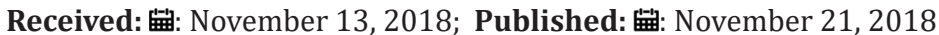

*Corresponding author: Mohammad Nazmul Hasan, Department of Green Bioengineering, Korea National University of Transportation Korea

\begin{abstract}
Oral immunization is considered the most convenient route of vaccination rather than any other route due to their abundance of facilities over the traditional vaccines. Gaining of mucosal immunity through oral vaccination can act as a protector against human to human or animal to human pathogenic transmission of diseases and also inhibit the pathogenic replication in the mucosal area which is the most preferable able route of microbe's penetration to the host blood circulation. Unfortunately, there are a handful of oral licensed vaccines in the market due to several drawbacks. Here, we tried to present a brief discussion on the various type of vaccines, oral vaccine delivering strategies and their current and future perspectives.
\end{abstract}

Abbreviations: OPV: Oral Polio Vaccine, VAPP: Vaccine-Associated Paralytic Polio, HBsAg: Hepatitis B Surface Antigen, HA: Hyaluronic Acid, HIV: Human Immunodeficiency Virus

\section{Introduction}

After the penetration of pathogenic microorganisms in our body, they (germs) instantly start to invade and multiply to increase their soldiers against body's immunity system resulting infection. Even though the immunity system has its own mechanisms to battle against such harmful germs by using its macrophages, T-lymphocytes as well as B-lymphocytes, maximum times fail in case of very harmful pathogens. According to the statistics of WHO in the year 2016, infectious diseases are still now causing severe mortality globally, especially in developing countries whereas infections are responsible for more than $30 \%$ of total death among top ten causes of human mortality [1]. Nowadays, researchers are more likely tend to develop vaccines to combat and eradicate deadly infectious diseases because of their extreme capability to fight against pathogens along with immunity. Moreover, vaccines have been used against various lethal infectious diseases from 1796 when Edward Jenner for the first time introduced smallpox vaccine till now with success [2]. Vaccines are usually a type of biological accumulation of antigens that take a step to activate adaptive immunity by mimicking an infection, thus, vaccines obviate harmful microorganisms as well as impede microbial evolution. The infection which is caused by vaccines usually doesn't cause illness of an individual, but his/her body be aware of this infection by treating this vaccine a threat and creates antibody and memory cells for further facing that infections causing microbes [3]. Immunization through vaccination is beneficial not only for vaccinated personnel but also his/her surrounding society by producing herb immunity [4]. Surprisingly, about $90 \%$ of total pathogens cross thin and easily vulnerable mucous membrane to invade cellular mechanisms via dealing with digestive, pulmonary and genitourinary systems due to the big surface area [5].

Hence, primary targeting site like a mucosal barrier for vaccine delivery would be the appropriate decision against infectioncausing pathogens. Once first defensive line will be strong only a few pathogens can cross the barrier. Researchers already proved that vaccine delivery targeting mucus membrane can easily produce mucus antibody IgA against pathogens [6] as well as increase cellular immune by secreting systemic antibody IgG $[7,8]$. Thus, when one site of the mucosal barrier will be protected, others site will be automatically protected through mucosal inner safeguard network [9]. Through oral vaccine delivery, it is possible to target the mucosal barrier rather than other routes of administration because of oral delivery of vaccines facilitate both IgA and IgG secretion in the body. Moreover, oral delivery is not the only potential for the best protective mechanisms but also it has its other merits like enhanced patient compliance, price-effectiveness, large-scale production, no harm and infection from the needle and so one. In contrast, IV/IM/SC administrated vaccines aren't able to stop pathogens in the mucosal barrier. Developing of oral 
vaccines is a novel approach by considering their challenges like extremely acidic condition, proteolytic enzymes, bile salts and their pharmacokinetics (ADME). Unfortunately, most of the potential vaccines are taken through injection whereas a handful number of vaccines are available for oral administration [10].

\section{Vaccine Types}

Scientists are trying from the time remember to develop various types of vaccines against harmful infectious pathogens with a similar function to teach the immune system how to combat against germs. For developing a vaccine against a specific virus infection, it is more important to consider both how germs attack the cells and how the response of our immune system to the germs. As vaccines enhance our cellular immunity, it is considered as the pre-treatment of pathogenic microorganisms before getting disease-causing microbes. However, according to the formulation and mechanism, vaccines can be categorized in the following folds.

Live Attenuated Vaccine: Among all types of vaccines, live attenuated vaccines are considered as the most successful and cost-effective [11]. Even, the history of vaccination was written with smallpox vaccine which was live attenuated. In addition, another attenuated vaccine such as the rabies vaccine was the first human vaccine that was made in a laboratory [12]. Live attenuated vaccine is usually developed by delivering the weakened virus to the human body. The delivered live attenuated viruses can result in asymptomatic infection without having a serious illness and harming another individual by means of spreading, thus, increase immune response and antibody production targeting to the specific delivered virus. The mechanisms behind the live attenuated vaccines why they usually don't create serious illness is, before introducing the selected live but weakened virus into the human body, they (virus) passage through in cell culture or animal embryo about 200 times to reproduce themselves. Among them, low virulence viruses are selected to grow in a large scale for preparation of a vaccine. Viruses which are low virulence they can easily replicate in a series of cell culture or in the chick embryo but not in the human host. Because of those weakened viruses are used to reproduce themselves in chick embryo or another media, hence, when they just change their hostile environment they cannot replicate or reproduce. Howsoever, the human body's immune system certainly attacks these weakened viruses by considering them as a threat and produce antibody to fight against them as well as keep some memory cells in the blood. In future, when same viruses come to cause infection memory cell and previously produced antibody catch and kill them [13].

Live attenuated vaccines facilitate lifetime safeguard against the virus by taking only a single or highly two doses of inoculation. These vaccines usually modulate all types of the immune signal as cellular immune mechanisms against viruses by secreting both IgA and IgG [14]. Not only that, live attenuated viruses confirm heterologous effects which are very potential to save an individual from non-specific infection [15]. Very rarely, attenuated viruses can reproduce in the human host which is more dangerous, hence, researchers should be concern about their responsibilities when planning to develop an attenuated vaccine [16]. In addition, the live attenuated vaccine is more likely cause adverse effects rather than any other vaccines because of its weakened but live virus. In a weak individual whose immunity system is not so strong like cancer or HIV patients to handle weakened live viruses, there are more possibilities of replication of viruses in the human body and cause infections [17]. For example, in the United States attenuated oral polio vaccine (OPV) is prohibited since 2000 according to the evidence of vaccine-associated paralytic polio (VAPP). National vaccination policy ruled the use of inactivated polio vaccine instead of OPV in 1997 when they found 9 persons were infected every year due to the administration of OPV [18].

Killed-Whole Cell Vaccines: To overcome various drawbacks of attenuated vaccines, killed-whole cell vaccines aroused which are known as inactivated vaccines. To produce an inactivated vaccine, researchers usually banish the targeted virus or bacteria and let them reproduce in culture. After that, those viruses are inactivated by heat, radiation or using chemicals like formalin or formaldehyde. Next, inactivated viruses are reproduced in large scale for preparation vaccine. Inactivated vaccines are more protective and static in comparison with the attenuated vaccine. In fact, whole killed vaccines cannot cause infections though it elicits a less strong immune response than live attenuated vaccines. The mechanism of actions behind their unique properties is when viruses or bacteria are inactivated in the laboratory they cannot replicate or reproduce within the human body (killed whole bacterial or viral organisms). In such conditions, though viral or bacterial organisms are inactivated they are intake this is because our body's immune system can easily recognize them and take further protective steps against such bacteria or viruses. Moreover, inactivated vaccines can be stored at the freeze-dried form and no need for refrigerating, so, in underdeveloped countries where need vaccination they can easily get it. Though inactivated vaccines are more safe with fewer side effects than attenuated vaccines, killed-whole cell vaccines exhibit less immune responses as well as antibodies. In addition, additional dose like booster doses is required to boost immune systems for long-term immunity in the case of inactivated vaccines [19].

Sub-Unit Vaccines: Unlike traditional live attenuated and/or inactivated vaccines in which live or inactivated germs were used, subunit vaccines contain the only antigen of a specific pathogen where there is no chance to get back as mutant [20]. Sometimes, the only epitope of antigens is used as a vaccine in which paratope (antigen-binding portion) of an antibody bind. In a subunit vaccine, 1 to20 antigenic part of microbes can be used which are more potential to exhibit a strong immune response [21]. To develop such efficacious, safer and more cost-effective vaccines with great pharmacological stability and to find out which combination of subunits provide strong immune responses, scientists test antigens of various potential subunit-like protein, sugar, and capsid. As only fragments of microbes are present in this type of vaccines, body's immunity system can attach to the key part of bacteria or viruses and finally destroy that's why every individual who has strong or less immunity can take this vaccine [22]. Though it provides 
strong immunity, additional booster doses are required to continue to prolong immunity. One of the major downsides of this vaccine is, no confirmation regarding forming immune memory cells. In comparison with live attenuated vaccines, they are less strong by means of immune responses [23]. Subunit vaccines can be further divided into the following categories.

Protein-based Subunit Vaccines: Protein-based vaccines are a type of subunit vaccines in which disease-causing proteins by means of antigen are used as a therapeutic tool. For developing such kind of vaccines researchers usually grow the bacteria or viruses in cell culture and then disintegrate those bacteria or viruses via using chemicals. Next, targeted proteins are collected for preparing vaccines. The proteins are isolated and purified before use as vaccines. Proteins are fragile in nature. Hence, there is more possibility to degrade by different $\mathrm{pH}$ factors of the gastrointestinal tract and/or to denature by proteolytic enzymes like trypsin, chymotrypsin, and pepsin. These brittle proteins have a various 3D structure that's why immune's antibody does not recognize them easily; resulting in non-specific antibody binding.

Polysaccharide Vaccines: In polysaccharides vaccines, a part of pathogenic polysaccharide capsule or pure cell membrane of sugar coat for example surface protein of polysaccharide is used. Sometimes bacterial or viral disease causing antigens are protected by their own sugar coat generally known as polysaccharides from body's natural defensive mechanisms [24]. This mechanism gives researchers two key points, first, develop such type of vaccines by which specific antibody can attach to the molecules in bacterial or viral polysaccharide capsules, second, bacterial protein is coated with the polysaccharide that's why infants and young children's immunity's system cannot recognize them. This is maybe for the improper development of the baby's immune system. However, the poor immunogenicity of children to polysaccharide vaccines downregulate their usages in the western or developed countries [25]. Though it is very easy to prepare anti-capsular antibody polysaccharide vaccine, it is used only for short time defensive mechanisms. As antibody generation is very slow with these vaccines, immunogenicity is also very poor, even, poor immune memory [26].

Conjugates Vaccine: The underdeveloped immune function of children that cannot work against polysaccharide antigens and the differences between mature and immature immune systems are one of the main causes to develop conjugate vaccines. The modern technology chemically linked a protein carrier (diphtheria or tetanus toxoids) from a different agent with microbial polysaccharide cell wall to enhance immunogenicity for a long time even in children [27]. The chemical linkage is the main reason why polysaccharideprotein conjugation vaccines are better rather than polysaccharide vaccines. Due to the introduction of the protein carrier with polysaccharide cell wall of microbes, an immature immune system can detect the polysaccharide coatings and immediately react to the bacterium [28]. To be specific, T cells of our immune system (both adult and child) first detect the protein carriers of polysaccharideprotein conjugation vaccines as well as alert B cells about the entrance of pathogenic antigens as foreign materials in our body. Then, plasma B cells produce a huge amount of antibody to destroy such threats [29]. The remaining memory B cells act as a defensive soldier against the microbes in future.

Toxoid Vaccines: Few bacteria like Clostridium tetani and Corynebacterium diphtheriae secret their poison called toxin in the bloodstream that can cause serious illness with severe symptoms. Inoculation against these bacteria is started base on their produced toxins. Scientists detoxified (inactivated) bacterial toxins by using formaldehyde solution with double distilled water or sometimes by heat or radiation. Finally, those inactivated toxins (toxoids) are used as vaccines. When bacterial toxoid is introduced in the human body, the natural immune system learns how to fight actual bacterial toxin [30]. Bacterial inactivated antigens (toxoids) are naturally innocuous because detoxified toxins cannot back at its virulent for even cannot multiply within the human host. Besides, toxoid vaccines are cost-effective, permanent and not prehensile to warm and damp [31]. Calcium and aluminum salts are used in the preparation of these vaccines to increase and strengthen their immune activity [27].

\section{Oral Vaccine Delivering Strategies}

There is no doubt that oral vaccine delivery is the most likely route among all routes of administration. Despite it, researchers usually concern about various physiological barriers and conditions of the human body and their interactions with vaccine therapeutics. For examples, vaccines particles first face the extreme acidic conditions of GIT as the first barrier, later on, the intestinal barriers like differences proteolytic enzymes (chymotrypsin, trypsin, bromelain, pain etc.), mucin barriers, intestinal retention and absorption of therapeutic particles also impede the successful oral vaccines delivery. In addition, generation of prolonging immunogenicity both in mucosal surface and systemic blood circulation depends on not only the successful oral delivery of vaccines but also their half-lives [32]. For low delivery strategies of vaccinogens by the oral route, high concentration or rapid delivery of vaccines particles are needed in comparison with other routes [33]. To overcome such drawbacks, researchers already proposed various oral delivery techniques as described below

Bacterial and/or Viral Vectors: In the promising field of recombinant biotechnology, using live vectors as either bacterial or viral are considered one of the most successful strategies in the account of oral vaccine delivery. Bacterial or viral vectors can cause upward trends of T-cell as well as mucosal and circular antibody promptness. Furthermore, immunogenicity works against both the antigens and their carrier by means of live vectors such as a double vaccine. Bacterial and viral vectors can replicate itself in the host, that's why more immunity produce against those vectors. Thus, these types of adenoviral systems can introduce a repetitive and sufficient amount of antigens to the internal immunity of the host. Nowadays, a series of live attenuated microbes are widely used for delivering of disease-causing antigens. For proposing a potential oral delivering strategy regarding the adenoviral system, researchers should diligently take into account the safety profile of live vectors 
due to their enhancing chances of causing virulence. An example would be, the use of Streptococcus pneumoniae as a bacterial vector for those who have the previous history of pneumonia. Not only that, live vectors are unable to protect vaccinogens in the $1.2 \mathrm{pH}$ of the stomach. Alginate coating of live vectors would be a suggestion for this unwanted situation. Target delivery of vaccine therapeutics with live vectors also possible through polymerization of vectors. Bacterial vectors recently used as an effective carrier of DNA vaccines facilitating enhanced physicochemical stability and prolonged immunity which will be very cost-effective [34].

On the other hand, S. typhi was successfully used as vector against hepatitis B diseases to deliver recombinant hepatitis B virus surface antigen in mice model [35] but no adequate response regarding immunogenicity was found in human [36]. In the second last decades, Tacket CO, et, al., proved that S. typhi can enhance serum antibody level against tetanus toxin (fragment C) [37]. Like recombinant bacterial vectors, a series of viral vectors are also using targeting various diseases. Among them, the adenoviral vectors are notably used worldwide. In the year 1979, Takafuji et al., described that live adenovirus can be used against respiratory tract infections [38]. Adenoviral based oral vaccine delivery is not only effective for human but also for veterinary used [39]. Unfortunately, in phase 1 clinical trial, one dose of hepatitis $B$ vaccination to healthy individuals did not found any hepatitis B antigen-specific antibody response [40]. Though live vectors can replicate in the intestine, enhance antigen absorption as well as target $\mathrm{M}$ cell in the follicleassociated epithelium of the Peyer patch, researchers suggest to the enteric coat of adenoviral vectors for preventing them from harsh gastric juice [41]. There is also another two type of viruses that are used as live viral vectors known as vaccinia and polioviruses [42] though using poliovirus as live vectors have their own limitation too.

Transgenic Plants: Traditionally plants are being used as therapeutics for thousands of years targeting various diseases [4345]. From many years, researchers were more tending to discover plant-based vaccine producing and delivering strategies. Now, various plants are used as transgenic plants facilitating needlefree oral vaccination to both infants and adults. Transgenic plantbased oral vaccine delivery facilitates a lot of advantages like locally cost-effective production, no need to use the refrigerator for storage and according to the statistics, the antigens present in the plant's parts like seeds or leaf are stable even in room temperature [46]. Transgenic plant-based inoculation can able to ensure mass vaccination which is very important for different developing countries. Early studies of transgenic plant-based vaccine production and delivery especially emphasized on potato and tobacco plants targeting various life-threatening diseases, for example, hepatitis B, malaria, HIV, and so ones. Among them, the Hepatitis B virus vaccine through transgenic plants achieved the most successful rate. An example would be, Mason SH et al., used tobacco leaves for the large-scale production of hepatitis b surface antigen (HBsAg) is almost similar to human serum's HBsAg [47].

They also proved that no obstacle would arrive regarding normal transcription or translation of antigen in transgenic plants like a tobacco plant. Another research suggested that regulating the expression of HBsAg using leaves of Nicotiana tabacum plant is also possible [48]. In this research article, Kumar GBS, et al., for the first time claimed about the expression of HBsAg in tobacco seeds. In addition, Richter and co-workers studied the ability of serum antibody production of transgenic potato plant derived antigen in a mice model as the preclinical studies that indicated a successive preliminary immunogenic response against delivered antigen [49]. Some researchers just took a gene responsible for Hepatitis B and inserted the gene into potato for developing a new era in vaccinology especially for poor countries [50]. Nevertheless, rice and corn also took an interest in oral vaccine delivery because these foods are used as baby food rather than any other food $[51,52]$. Vaccination with transgenic plant-derived antigen is more acceptable and reliable rather than any other sources.

Lipid-based Vectors: Liposome contains lipid bilayer systems in which they can imbibe both hydrophilic nucleic acid, vaccine's antigen, and hydrophobic drugs at a time. Through surface modification, they can facilitate target delivery of antigen to m-cell and protect antigen from extreme physiological $\mathrm{pH}$. Modified liposomal systems with vaccinogens, adjuvants, and targeting moiety are increased oral vaccinology even though they have limited conduction to gut-assisted lymphoid tissue and systemic absorption. From the starting history when Childers and colleagues proved liposome-based secreting IgA secretion [53], after that, a lot of data indicated their ability to enhance mucosal immunity like Chaicumpa et al., compared the liposomal-based cholera antigenmediated immunogenicity with naked antigen resulting in a novel approach of oral cholera vaccine delivery via the liposomal system [54]. Fortunately, lipid-based vectors have more interaction ability with intestinal m cells which is the key transporting components of oral vaccine delivery in comparison with any other cell like mucussecreting goblet cell or other enterocytes. M cells are responsible for both receptor-mediated or non-receptor mediated transportation of liposome vehicles [55,56]. The introduction of the liposomal system in oral vaccine delivery from the end of the last decades presented various animal studies as well as clinical data but their superabundant vaccination effects are still in major questions.

Nanoparticle-based Transportation: Oral vaccine delivery using nanoparticles are usually considered the most successful strategy among all transportation systems. Nanoparticles are usually biocompatible and biodegradable in nature that can protect vaccinogens from acidic ph and enzymatic degradation. Nanoparticles like Betaglucan itself has the ability to target M cell. In addition, surface-modified nanoparticles with GRGDS or CKS9 like $M$ cell targeting moiety can able to target wise delivery of antigens into M-cell [24]. On the other hand, positively charge nanoparticles can easily target negatively charged $\mathrm{M}$ cell. Thus, targeting ability of nanoparticles based vaccine delivery system is the most convenient than any other systems. To be specific PLG, PLGA, PEG and PELA are the common polymeric nanoparticles for vaccine delivery.

Denaturation of these nanoparticles by various physiological enzymes may decrease their potentiality. Protamine is another impressive nanoparticle that can conjugate with antigen through 
electrostatic interactions and also protamine either alone or in combination with antigen can be localized into nuclease. Watersoluble chitosan and HA (hyaluronic acid) are another novel postulant under this system. Thousands of studies suggested their acceptability in oral vaccine delivery. Hence, nanoparticle-based transportation of viral antigen for convenient oral delivery would be the best answer to those arising questions related to major obstacles of oral vaccine delivery strategy. There are also a few

Table 1: In clinical Trials.

\begin{tabular}{|c|c|c|c|}
\hline Phase & Name of Vaccine & Targeting disease & Manufacturers \\
\hline II & Cholera Grade & HIV & AVANT Immunotherapeutic, USA \\
\hline II \& I & RV3-BB & Rotavirus & GlaxoSmithKline, UK \\
\hline IIb & LATTE-2 & $\mathrm{HCV}$ & GlaxoSmithKline, UK \\
\hline III, II \& I & ORV 116E & Rotavirus & SAS, India \\
\hline
\end{tabular}

Table 2: Licensed Vaccines.

\begin{tabular}{|c|c|c|}
\hline Name of Vaccine & Targeting disease & Manufacturers \\
\hline Vivotif & Salmonella typhi & Paxvax, USA \\
\hline Dukoral & \multirow[t]{4}{*}{ Vibro Cholera } & VALNEVA, France \\
\hline Envichol & & Eubiologics, South Korea \\
\hline Cholvax & & Incepta, Bangladesh \\
\hline Shanchol & & SANOFI, France \\
\hline Biopolio B1/3 & Types 1, 2, \& 3 attenuated poliomyelitis viruses & Bharat Biotech, India \\
\hline RotaTeq & \multirow[t]{2}{*}{ Rota Virus } & MSD, USA \\
\hline Rotarix & & GlaxoSmithKline, UK \\
\hline
\end{tabular}

\section{Conclusion and Future Perspectives}

Vaccine delivery techniques have improved a lot with the pace of time even though there are a few vaccines available in the market so far. Oral vaccine delivery is most convenient for the patience and higher efficiency of vaccines can be achieved by oral gavage compare to other delivery routes. So far, oral vaccines are delivered, targeting the M-Cells in small intestine due to better uptake performance. But number of M-cells are very few in the human body, thus it is important to find another mechanism to delivery oral vaccine successfully. In future, researchers might target other cells rather than M-cells and there is ongoing work based on targeting epithelial cells and so on. Several oral vaccines, e.g., hepatitis C, HIV (Human Immunodeficiency Virus) are on Phase II trials and can bring success in oral delivery vaccines in future.

\section{References}

1. (2016) The top ten causes of death, WHO.

2. Plotkin SL, S Plotkin (2008) A short history of vaccination. In: S Plotkin, WA Orenstein, PA Offit (Eds.)., Elsevier-Saunders, Phildadelphia, USA.

3. National Institute of Allergy and Infectious Diseases, Understanding Vaccines.

4. M Doherty, P Buchy, B Standaert, C Giaquinto, D Prado Cohrs (2016) Vaccine impact: benefits for human health. Vaccine 34(52): 6707-6714.

5. J Holmgren, C Czerkinsky (2005) Mucosal immunity and vaccines. Nat Med 11: S45-S53. of strategies like immune stimulating complexes and virus-like particles which also influence researchers to develop a new oral vaccine to overcome traditional vaccine strategies.

\section{Progression of Oral Vaccines}

Oral vaccines have been developed emergingly from the first application till now. Several oral vaccines are available in the market and a few are in clinical trials. A list of oral vaccines progression is given here for research perusal $[1,27,42,56,57]$ (Tables $1 \& 2$ ). 
16. Benn Christine S, Netea Mihai G, Selin Liisa K, Aaby Peter (2013) A small jab -a big effect: nonspecific immunomodulation by vaccines. Trends in Immunology Elsevier 34(9): 431-439.

17. Shimizu H, Thorley B, Paladin FJ, Schulz M, Lukanova A, et al. (2004) Circulation of type 1 vaccine-derived poliovirus in the Philippines in 2001. J Virol 78(24): 13512-13521.

18. LN Alexander, JF Seward, TA Santibanez, MA Pallansch, OM Kew, et al. (2004) Vaccine policy changes and epidemiology of poliomyelitis in the United States. JAMA 292: 1696-1701.

19. NA Summerton, RW Welch, L Bondoc, HH Yang, B Pleune, et al. (2010) Toward the development of a stable, freeze-dried formulation of Helicobacter pylori killed whole cell vaccine adjuvanted with a novel mutant of Escherichia coli heat-labile toxin. Vaccine 28(5): 1404-1411.

20. Baxter D (2007) Active and passive immunity, vaccine types, excipients and licensing. Occup. Med 57(8): 552-556.

21. Vaccine Healthcare Centers Network.

22. Scott C (2004) Classifying vaccines: From cowpox to the cutting edge. Bio Process Int 2: 14-23.

23. Vartak A, Sucheck SJ (2016) Recent Advances in Subunit Vaccine Carriers. Vaccines 4(2): 12.

24. Chi Jen Lee, Lucia H Lee, MD Kaio Koizumi (2002) Polysaccharide Vaccines for Prevention of Encapsulated Bacterial Infections: Part 1 Infect Med 19(3): 127-133.

25. Austrian R (1985) Polysaccharide vaccines. Annales de l'Institut Pasteur / Microbiologie 136(3): 295-307.

26. Lesinski GB, Westerink MA (2001) Vaccines against polysaccharide antigens. Curr Drug Targets Infect Disord 1(3): 325-334.

27. (2013) NIAID, Types of vaccines, HHS (Edn.).

28. Peeters C, Patrick R Lagerman, Odo de Weers, Lukas A Oomen (2003) Preparation of Polysaccharide-Conjugate Vaccines. Methods Mol Med 87: 153-174.

29. Bhav Patel (2014) Differences between polysaccharide and polysaccharide-protein conjugate vaccines Science Nutshell.

30. D Baxter (2007) Active and passive immunity, vaccine types, excipients and licensing. Occup Med (Lond) 57(8): 552-556.

31. Avila L, Cascone O, Biscoglio M, Fingermann M (2018) An effective, simple and low-cost pretreatment for culture clarification in tetanus toxoid production. Prep Biochem Biotechnol 28: 1-7.

32. De Smet, , Allais L, Cuvelier CA (2014) Recent advances in oral vaccine development: yeast-derived $\beta$-glucan particles. Human vaccines \& immunother 10(5): 1309-1318.

33. Periwal SB, Kourie KR, Ramachandran N, Blakeney SJ, DeBruin S, et al. (2003) A modified cholera holotoxin CT-E29H enhances systemic and mucosal immune responses to recombinant Norwalk virus-virus like particle vaccine. Vaccine 21(5-6): 376-385.

34. Medina E, Guzman CA (2001) Use of live bacterial vaccine vectors for antigen delivery: potential and limitations. Vaccine 19(13-14): 15731580.

35. Xiang R, Lode HN, Chao TH, J Michael Ruehlmann, Carrie S Dolman, et al. (2000) An autologous oral DNA vaccine protects gainst murine melanoma. Proc Natl Acad Sci USA 97(10): 5492-5497.

36. Gomez Duarte OG, Lucas B, Yan ZX, Panthel K, Haas R, et al. (1998) Protection of mice against gastric colonization by Helicobacter pylori by single oral dose immunization with attenuated Salmonella typhimurium producing urease subunits A and B. Vaccine 16(5): 460-471.

37. Tacket CO, Kelly SM, Schodel F, Losonsky G, Nataro JP, et al. (1997) Safety and immunogenicity in humans of an attenuated Salmonella typhi vaccine vector strain expressing plasmid-encoded hepatitis B antigens stabilized by the Asd-balanced lethal vector system. Infect Immun 65(8): 3381-3385.

38. Tacket CO, Galen J, Sztein MB, Losonsky G, Wyant TL, et al. (2000) Safety and immune responses to attenuated Salmonella enterica serovar typhi oral live vector vaccines expressing tetanus toxin fragment C. Clin Immunol 97(2): 146-153.

39. Takafuji ET, Gaydos JC, Allen RG, Top JFH (1979) Simultaneous administration of live, enteric-coated adenovirus types 4,7, and 21 vaccines: Safety and immunogenicity. J Infect Dis 140(1): 48-53.

40. Tuboly T, Nagy E (2001) Construction and characterization of recombinant porcine adenovirus serotype 5 expressing the transmissible gastroenteritis virus spike gene. J Gen Virol 82: 183-190.

41. Tacket CO, Losonsky G, Lubeck MD, Davis AR, Mizutani S, et al. (1992) Initial safety and immunogenicity studies of an oral recombinant adenohepatitis B vaccine. Vaccine 10(10): 673-676.

42. Deal C, Pekosz A, Ketner G (2013) Prospects for oral replicating adenovirus-vectored vaccines. Vaccine 31(32): 3236-3243.

43. Rolph MS, Ramshaw IA (1997) Recombinant viruses as vaccines and immunological tools. Curr Opin Immunol 9(4): 517-524.

44. SM Shatil Shahriar, Mohammed Rahmatullah (2015) Evaluation of Antihyperglycemic Activity of A Polyherbal Formulation Diabeto-VLC, WJPR 5(1): 191-198.

45. SM Shatil Shahriar, Jannatul Ferdoes Shoma, Mohammed Rahmatullah, Oral Glucose Tolerance Test (Ogtt) With Whole Plants of Alocasia Fornicata Roxb. WJPR 6(12): 48-53.

46. SM Shatil Shahriar, Mohammed Rahmatullah, Oral Glucose Tolerance Tests in Mice with a Polyherbal Formulation Diabeto-MLF 5(1): 199-206.

47. Lamphear BJ, Streatfield SJ, Jilka JM, Brooks CA, Barker DK, et al. (2002) Delivery of subunit vaccines in maize seed. J Control Release 85: 169-80.

48. Mason HS, Lam DM, Arntzen CJ (1992) Expression of hepatitis B surface antigen in transgenic plants. Proc Natl Acad Sci U S A 89(24): 1174511749.

49. Kumar GS, Srinivas L, Ganapathi T (2006) Plant Cell Tiss Organ Cult 84: 315.

50. Liz J Richter, Yasmin Thanavala, Charles J Arntzen, Hugh S Mason (2000) Production of hepatitis B surface antigen in transgenic plants for oral immunization, Nature Biotechnology 18(11): 1167-1171.

51. Roxanne Khamsi (2005) Potatoes pack a punch against hepatitis B, Published online Nature.

52. Webster D, Thomas M, Strugnell R, Dry IB, Wesselingh SL (2002) Appetising solutions; an edible vaccine for measles. Med J 176: 434-437.

53. Streatfield SJ, Lane JR, Brooks CA, Barker DK, Poage ML, et al. (2003) Corn as a production system for human and animal vaccines. Vaccine 21: 812-815.

54. Childers NK, Michalek SM, Pritchard DG, Mc Ghee JR (1990) Mucosal and systemic responses to an oral liposome-streptococcus mutans carbohydrate vaccine in humans. Reg Immunol 3: 289-296.

55. Chaicumpa W, Chongsa nguan $\mathrm{M}$, Kalambaheti T, Wilairatana $\mathrm{P}$, Srimanote P, et al. (1998) Immunogenicity of liposome-associated and refined antigen oral cholera vaccines in Thai volunteers. Vaccine 16: 678-684.

56. Harsløf T, Langdahl BL (2016) New horizons in osteoporosis therapies. Curr Opin Pharm 28: 38-42.

57. Julia E, Vela Ramirez, Lindsey A Sharpe, Nicholas A Peppas (2017) Current state and challenges in developing oral vaccines. Adv Drug Deliv Rev 114: 116-131. 
ISSN: 2574-1241

DOI: $10.26717 / B J S T R .2018 .11 .002075$

Mohammad Nazmul Hasan. Biomed J Sci \& Tech Res

CC (i) This work is licensed under Creative

Submission Link: https://biomedres.us/submit-manuscript.php

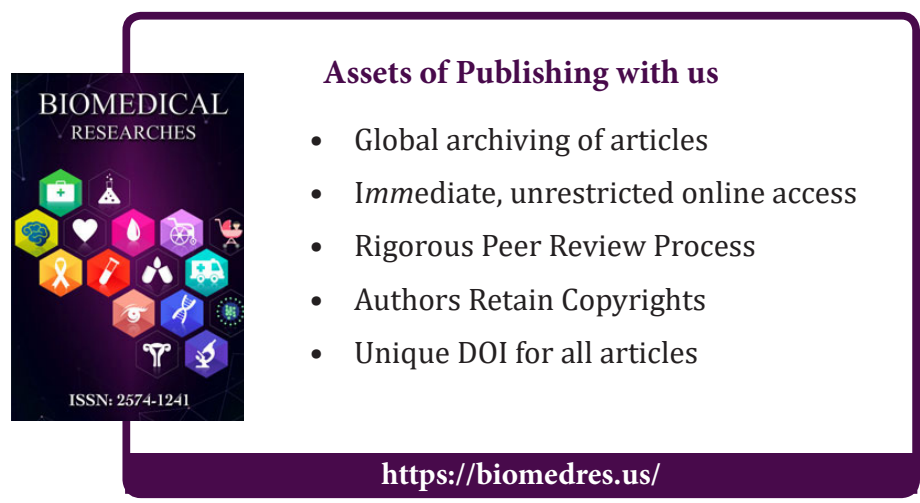

